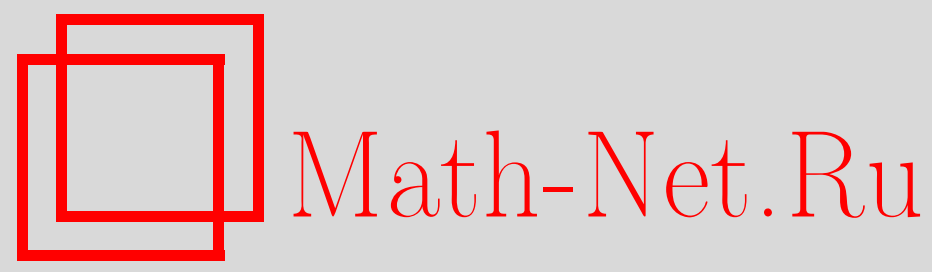

Ю. С. Харин, А. И. Петлицкий, Идентификация двоичной цепи Маркова $s$-го порядка с $r$ частичными связями при наличии аддитивных искажений, Дискрет. матем., 2010, том 22, выпуск 4, 138-155

DOI: https://doi.org/10.4213/dm1124

Использование Общероссийского математического портала Math-Net.Ru подразумевает, что вы прочитали и согласны с пользовательским соглашением http://www . mathnet.ru/rus/agreement

Параметры загрузки:

IP : 3.91 .87 .62

26 апреля 2023 г., $16: 32: 38$ 


\title{
Идентификация двоичной цепи Маркова $s$-го порядка с $r$ частичными связями при наличии аддитивных искажений
}

\author{
( 2010 г. .С. Харин, А. И. Петлицкий
}

\begin{abstract}
Статья посвящена решению актуальной задачи идентификации (оцениванию параметров и проверке гипотез) для двоичной цепи Маркова ЦМ $(s, r)$ при наличии аддитивных искажений.

Работа выполнена в рамках Государственной программы фундаментальных исследований Республики Беларусь «Математические модели».
\end{abstract}

\section{1. Введение}

Цепи Маркова высокого порядка имеют широкое применение при исследовании зависимостей в стохастических последовательностях [1-14]. Однако число параметров (вероятностей переходов) цепи Маркова $s$-го порядка растет экспоненциально при увеличении $s$, что усложняет идентификацию этой модели на практике. Для снижения вычислительной сложности разрабатываются малопараметрические модели цепей Маркова высокого порядка: бинарная авторегрессия [5], модель Джекобса-Льюиса [6], MTD-модель Рафтери [7], цепь Маркова переменного порядка [8], цепь Маркова $s$-го порядка с $r$ частичными связями $[9,10]$, для которой принято использовать краткое обозначение цМ $(s, r)$.

В приложениях возникает необходимость в так называемых усложненных моделях цепи Маркова высокого порядка: искаженная цепь Маркова [11], модель скрытой цепи Маркова [12], модель двойной цепи Маркова [13]. Ряд работ посвящен статистическому анализу цепей Маркова при наличии искажений $[11,14]$. Общее условие сохранения марковости преобразования независимых двоичных последовательностей конечных однородных цепей Маркова первого порядка представлено в [14]. В [11] проведен анализ суммы цепи Маркова первого порядка и авторегрессионного процесса (в поле действительных чисел). Результаты исследования цепей Маркова при наличии искажений могут быть использованы для улучшения статистических свойств выходных последовательностей генераторов случайных и псевдослучайных последовательностей.

Данная статья посвящена решению актуальной задачи идентификации (оцениванию параметров и проверке гипотез) для двоичной цепи Маркова ЦМ $(s, r)$ при наличии аддитивных искажений. 


\section{2. Двоичная цепь Маркова с частичными связями и ее вероятностно-статистические свойства}

Пусть $A=\{0,1\} ; J_{i}^{k}=\left(j_{i}, j_{i+1}, \ldots, j_{k}\right) \in A^{k-i+1}-$ цепочка из $k-i+1$ знаков последовательности $\left\{j_{n}\right\}, k \geqslant i ;\left\{x_{t}\right\}$ - однородная цепь Маркова $s$-го порядка с множеством состояний $A$ и вероятностями одношаговых переходов

$$
p_{J_{1}^{s+1}}=\mathbf{P}\left\{x_{t+s}=j_{s+1} \mid x_{t+s-1}=j_{s}, \ldots, x_{t}=j_{1}\right\}, J_{1}^{s+1} \in A^{s+1}, t \in \mathbf{N}=\{1,2, \ldots\} .
$$

Цепь Маркова $s$-го порядка называется цепью с $r$ частичными связями $[9,10]$, если ее вероятности одношаговых переходов имеют вид

$$
p_{j_{1}, \ldots, j_{s}, j_{s+1}}=q_{m_{1}^{0}, \ldots, j_{m_{r}^{0}}, j_{s+1}}, \quad J_{1}^{s+1} \in A^{s+1},
$$

где $r \in\{1,2, \ldots, s\}$ - число связей; $M_{r}^{0}=\left(m_{1}^{0}, m_{2}^{0}, \ldots, m_{r}^{0}\right) \in \mathrm{M}$ - целочисленный $r$-вектор с упорядоченными компонентами $1=m_{1}^{0}<m_{2}^{0}<\ldots<m_{r}^{0} \leqslant s$, называемый шаблоном связей, $\mathrm{M}-$ множество всевозможных таких векторов; $Q=\left(q_{J_{1}^{r+1}}\right)_{J_{1}^{r+1} \in A^{r+1}}$ - $(r+1)$-мерная стохастическая матрица, то есть матрица, элементы которой неотрицательны и $\sum_{j_{r+1} \in A} q_{J_{1}^{r+1}}=1, J_{1}^{r} \in A^{r}$.

Соотношение (1) означает, что вероятность перехода в состояние $j_{s+1}$ зависит не от всех $s$ предыдущих состояний $j_{1}, \ldots, j_{s}$, а лишь от $r$ избранных состояний $j_{m_{1}^{0}}, \ldots, j_{m_{r}^{0}}$. Таким образом, вместо $N^{s}(N-1)$ параметров матрица вероятностей одношаговых переходов ЦМ $(s, r)$ полностью определяется $N^{r}(N-1)+\min \{r-1, s-r\}$ параметрами $Q$, $M_{r}^{0}$ (при $r-1>s-r$ шаблон связей однозначно задается с помощью $s-r$ элементов, не входящих в него). Заметим, что если $r=s$, то получаем цепь Маркова $s$-го порядка [2], то есть ЦМ $(s, s)=$ ЦМ $(s)$.

Теорема 1 ([10]). Цепь Маркова s-го порядка с $r$ частичными связями, определяемая (1), является эргодической тогда и только тогда, когда найдется $i \in \mathbf{N}$ такое, что

$$
\min _{J_{1}^{s}, J_{s+i+1}^{2 s+i} \in A^{s}} \sum_{J_{s+1}^{s+i} \in A^{i}} \prod_{k=1}^{s+i} q_{j_{k+m_{1}^{0}-1}^{0}, \ldots, j_{k+m_{r}^{0}-1}^{0}, j_{k+s}}>0 .
$$

Следствие 1 ([10]). Если $\min _{J_{1}^{r+1} \in A^{r+1}} q_{J_{1}^{r+1}}>0$, то ЦМ $(s, r)$ является эргодической.

В дальнейшем будем предполагать, что ЦМ $(s, r)$ является стационарной $[2,18]$, то есть для нее выполнено условие эргодичности и ее начальное распределение вероятностей $\left(\pi_{J_{1}^{s}}\right)_{J_{1}^{s} \in A^{s}}$ совпадает со стационарным $\left(\pi_{J_{1}^{s}}^{*}\right)_{J_{1}^{s} \in A^{s}}$.

Примем еще несколько обозначений: $X_{1}^{n}=\left(x_{1}, x_{2}, \ldots, x_{n}\right)$ - реализация цепи Маркова ЦМ $(s, r)$ длины $n ; F\left(J_{1}^{s} ; M_{r}\right)=\left(j_{m_{1}}, j_{m_{2}}, \ldots, j_{m_{r}}\right)-$ функция, называемая селектором $r$-го порядка, $J_{1}^{s} \in A^{s}, M_{r} \in \mathrm{M} ; \delta_{J_{1}^{s}, K_{1}^{s}}=\prod_{i=1}^{s} \delta_{j_{i}, k_{i}}-$ символ Кронекера для $J_{1}^{s}, K_{1}^{s} \in A^{s}$;

$$
v_{J_{1}^{r+1}}\left(X_{1}^{n} ; M_{r}\right)=\sum_{t=1}^{n-s} \delta_{F}\left(X_{t}^{t+s-1} ; M_{r}\right), J_{1}^{r} \delta_{x_{t+s}, j_{r+1}}, \quad J_{1}^{r+1} \in A^{r+1},
$$


- частотные статистики для шаблона $M_{r} \in \mathrm{M} ;\left\{\pi_{J_{1}^{s}}^{*}: J_{1}^{s} \in A^{s}\right\}$ - стационарное распределение вероятностей ЦМ $(s, r)$;

$$
\begin{aligned}
\mu_{J_{1}^{r+1}}\left(M_{r}\right) & =\mathbf{P}\left\{F\left(X_{t}^{t+s-1} ; M_{r}\right)=J_{1}^{r}, x_{t+s}=j_{r+1}\right\} \\
& =\sum_{K_{1}^{s} \in A^{s}} \delta_{F\left(K_{1}^{s} ; M_{r}\right), J_{1}^{r} \pi_{K_{1}^{s}}^{*} p_{K_{1}^{s}, j_{r+1}}, \quad J_{1}^{r+1} \in A^{r+1},}
\end{aligned}
$$

- распределение вероятностей $(r+1)$-грамм для шаблона связей $M_{r} \in \mathrm{M}$;

$$
\hat{\mu}_{J_{1}^{r+1}}\left(M_{r}\right)=v_{J_{1}^{r+1}}\left(X_{1}^{n} ; M_{r}\right) /(n-s)
$$

- частотная оценка вероятности $\mu_{J_{1}^{r+1}}\left(M_{r}\right)$, которая является несмещенной и состоятельной в среднеквадратическом оценкой при $n \rightarrow \infty$ [10]. Условимся, что если вместо какого-то индекса стоит точка, то это означает суммирование по всем возможным значениям этого индекса:

$$
\mu_{J_{1}^{r}} \cdot\left(M_{r}\right)=\sum_{j_{r+1} \in A} \mu_{J_{1}^{r+1}}\left(M_{r}\right) .
$$

Если шаблон $M_{r}^{0}$ известен, то при $n \rightarrow \infty$ несмещенная и состоятельная в среднеквадратическом оценка для матрицы $Q$ имеет вид [10]

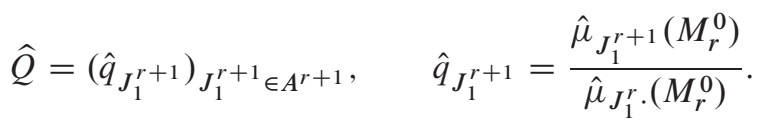

Состоятельная оценка для шаблона $M_{r}^{0}$ имеет вид [10]

$$
\widehat{M}_{r}=\arg \min _{M_{r} \in \mathrm{M}} \hat{H}\left(M_{r}\right),
$$

где

$$
\hat{H}\left(M_{r}\right)=-\sum_{J_{1}^{r+1} \in A^{r+1}} \hat{\mu}_{J_{1}^{r+1}}\left(M_{r}\right) \ln \left(\hat{\mu}_{J_{1}^{r+1}}\left(M_{r}\right) / \hat{\mu}_{J_{1}^{r}} \cdot\left(M_{r}^{0}\right)\right)
$$

- подстановочная оценка условной энтропии будущего символа $x_{t+s} \in A$ относительно прошлого, заданного селектором $F\left(X_{t}^{t+s-1} ; M_{r}\right) \in A^{r}, M_{r} \in \mathrm{M}$.

Состоятельные оценки $\hat{s}, \hat{r}$ для параметров $s, r\left(s_{-} \leqslant s \leqslant s_{+}, r_{-} \leqslant r \leqslant r_{+} \leqslant s_{+}\right)$ построены в [15] из условия минимума байесовского информационного функционала [16]

$$
\operatorname{BIC}(s, r)=2(n-s) \hat{H}\left(\widehat{M}_{r}\right)+N^{r}(N-1) \ln (n-s) .
$$

\section{3. Вероятностно-статистические свойства двоичной ЦМ $(s, r)$ при наличии аддитивных искажений}

Под двоичной цепью Маркова с частичными связями при наличии аддитивных искажений понимается наблюдаемый временной ряд [17]

$$
y_{t}=x_{t} \oplus \xi_{t}, \quad t \in \mathbf{N},
$$

где $x_{t} \in A-$ ненаблюдаемая двоичная ЦМ $(s, r), \xi_{t} \in A-$ ненаблюдаемая последовательность независимых в совокупности, одинаково распределенных двоичных случайных величин, $\mathbf{P}\left\{\xi_{t}=0\right\}=1-\mathbf{P}\left\{\xi_{t}=1\right\}=p>1 / 2 ;\left\{x_{t}\right\},\left\{\xi_{t}\right\}$ независимы. 
Отметим, что соотношение (7) определяет скрытую цепь Маркова, которая при $r=s=1$, то есть в случае простой цепи Маркова $x_{t}$, исследована в [12]. Заметим также, что если $p=1 / 2$, то $y_{t}$ - равновероятная случайная последовательность Бернулли [4]; в этом случае невозможно оценить параметры цепи Маркова с частичными связями $x_{t}$ по наблюдаемой реализации $y_{t}$.

Пусть $Y_{1}^{n}=\left(y_{1}, y_{2}, \ldots, y_{n}\right) \in A^{n}-$ наблюдаемая реализация длины $n$ для двоичной ЦМ $(s, r)$ при наличии аддитивных искажений.

Лемма 1. Для модели (7) функичия правдоподобия параметров $Q$ и р имеет вид

$$
L(Q, p)=\sum_{J_{1}^{n} \in A^{n}} p^{n}\left(\frac{1-p}{p}\right)^{w\left(J_{1}^{n} \oplus Y_{1}^{n}\right)}\left(\pi_{J_{1}^{s}} \prod_{i=1}^{n-s} q_{F}\left(J_{i}^{i+s-1} ; M_{r}^{0}\right), j_{i}+s\right),
$$

где $\left\{\pi_{J_{1}^{s}}: J_{1}^{s} \in A^{s}\right\}-$ начальное распределение вероятностей, $w(\cdot)-$ вес Хэмминга.

Доказательство. Вид функции правдоподобия получается прямым вычислением:

$$
L(Q, p)=\sum_{J_{1}^{n} \in A^{n}} \mathbf{P}\left\{X_{1}^{n}=J_{1}^{n}\right\} \mathbf{P}\left\{\Xi_{1}^{n} \oplus X_{1}^{n}=Y_{1}^{n} \mid X_{1}^{n}=J_{1}^{n}\right\} .
$$

Прямое вычисление значения функции $L(Q, p)$ при наблюдении временного ряда длины $n$ требует произвести $O\left(n 2^{n}\right)$ операций, что весьма затруднительно. Эта трудность также возникает в работах $[12,13]$.

Обозначим

$$
b_{K_{1}^{r+1}}\left(M_{r}\right)=\mathbf{P}\left\{F\left(Y_{t}^{t+s-1} ; M_{r}\right)=K_{1}^{r}, y_{t+s}=k_{r+1}\right\}, \quad K_{1}^{r+1} \in A^{r+1},
$$

распределение вероятностей $(r+1)$-граммы искаженной цепи Маркова для шаблона связей $M_{r} \in \mathrm{M}$.

Лемма 2. Если $x_{t}$ - стациионарная ичепь Маркова с частичныли связями, то справедливы соотношения

$$
b_{K_{1}^{r+1}}\left(M_{r}\right)=\sum_{J_{1}^{r+1} \in A^{r+1}} \mu_{J_{1}^{r+1}}\left(M_{r}\right) p^{r+1-w\left(J_{1}^{r+1} \oplus K_{1}^{r+1}\right)}(1-p)^{w\left(J_{1}^{r+1} \oplus K_{1}^{r+1}\right)},
$$

где $K_{1}^{r+1} \in A^{r+1}$.

Доказательство. С учетом (3), (7), (8), прямым вычислением вероятностей получаем требуемый результат.

Следствие 2. Если $p=1 / 2$ (искажающая последовательность чисто случайна), то $b_{K_{1}^{r+1}}\left(M_{r}\right)=2^{-(r+1)}$ для любых $K_{1}^{r+1} \in A^{r+1}, M_{r} \in \mathrm{M}$; если $p=1$ (искажения отсутствуют), по $b_{K_{1}^{r+1}}\left(M_{r}\right)=\mu_{K_{1}^{r+1}}\left(M_{r}\right)$.

Лемма 3. В условиях леммы 2 распределение вероятностей $(r+1)$-граммы для искаженной ЦМ $(s, r)$ не дальше (в евклидовой метрике) от равномерного, чем распределение $(r+1)$-граммы для неискаженной ЦМ $(s, r)$ :

$$
\sum_{J_{1}^{r+1} \in A^{r+1}}\left(b_{J_{1}^{r+1}}\left(M_{r}\right)-2^{-(r+1)}\right)^{2} \leqslant \sum_{J_{1}^{r+1} \in A^{r+1}}\left(\mu_{J_{1}^{r+1}}\left(M_{r}\right)-2^{-(r+1)}\right)^{2} .
$$


Доказательство. Примем матричные обозначения

$$
\begin{aligned}
\mu & =\left(\mu_{J_{1}^{r+1}}\left(M_{r}\right)\right) \in \mathbf{R}^{2^{r+1}}, \quad b=\left(b_{J_{1}^{r+1}}\left(M_{r}\right)\right) \in \mathbf{R}^{2^{r+1}}, \\
\tau & =\left(2^{-(r+1)}\right) \in \mathbf{R}^{2^{r+1}}, \quad V=\left(v_{J_{1}^{r+1}, K_{1}^{r+1}}\right) \in \mathbf{R}^{2^{r+1} \times 2^{r+1}}, \\
v_{J_{1}^{r+1}, K_{1}^{r+1}} & =p^{r+1-w\left(J_{1}^{r+1} \oplus K_{1}^{r+1}\right)}(1-p)^{w\left(J_{1}^{r+1} \oplus K_{1}^{r+1}\right)},
\end{aligned}
$$

причем $V=V^{\mathrm{T}}-$ бистохастическая матрица, $V \tau=\tau, \lambda_{\max }(V)=1-$ максимальное характеристическое число матрицы $V$. Тогда, используя лемму 2 и свойства характеристических чисел [22], получаем цепочку неравенств

$$
\begin{aligned}
\sum_{J_{1}^{r+1} \in A^{r+1}}\left(b_{J_{1}^{r+1}}\left(M_{r}\right)-2^{-(r+1)}\right)^{2} & =\|b-\tau\|^{2}=\|V \mu-\tau\|^{2}=\|V(\mu-\tau)\|^{2} \\
= & (\mu-\tau)^{\mathrm{T}} V^{2}(\mu-\tau) \leqslant \lambda_{\max }\left(V^{2}\right)\|\mu-\tau\|^{2} \\
= & \lambda_{\max }(V)\|\mu-\tau\|^{2}=\|\mu-\tau\|^{2} \\
= & \sum_{J_{1}^{r+1} \in A^{r+1}}\left(\mu_{J_{1}^{r+1}}\left(M_{r}\right)-2^{-(r+1)}\right)^{2} .
\end{aligned}
$$

В дальнейшем при доказательстве некоторых утверждений будем использовать представление ЦМ $(s, r)$ в виде цепи Маркова первого порядка с расширенным пространством состояний $A^{s}$ и вероятностями переходов $p_{I_{1}^{s}, L_{1}^{s}}^{*}=\delta_{I_{2}^{s}, L_{1}^{s-1}} p_{I_{1}^{s}, l_{s}}, I_{1}^{s}, L_{1}^{s} \in A^{s}$. Пусть $p_{I_{1}^{s}, L_{1}^{s}}^{*(k)}-$ вероятность перехода за $k$ шагов из состояния $I_{1}^{s}$ в $L_{1}^{s}$;

$$
c_{I_{1}^{s}, L_{1}^{s}}=\sum_{k=0}^{\infty}\left(p_{I_{1}^{s}, L_{1}^{s}}^{*(k)}-\pi_{L_{1}^{s}}^{*}\right), \quad I_{1}^{s}, L_{1}^{s} \in A^{s}
$$

(если ЦМ является эргодической, то этот ряд сходится [14]); $M_{r+1}=\left(M_{r}, s+1\right)$;

$$
\tilde{b}_{K_{1}^{r+1}}\left(M_{r}\right)=\frac{{ }^{{ }_{K_{1}^{r+1}}\left(Y_{1}^{n} ; M_{r}\right)}}{n-s}=\frac{1}{n-s} \sum_{t=1}^{n-s} \delta_{F\left(Y_{t}^{t+s-1} ; M_{r}\right), K_{1}^{r}} \delta_{y_{t+s}, k_{r+1}}
$$

- частотная оценка вероятности $b_{K_{1}^{r+1}}\left(M_{r}\right), K_{1}^{r+1} \in A^{r+1}$;

$$
\varkappa_{J_{1}^{r+1}, K_{1}^{r+1}}=\sum_{A(s, r)} \delta_{F\left(I_{1}^{s} ; M_{r}\right), J_{1}^{r}} \delta_{F\left(L_{1}^{s} ; M_{r}\right), K_{1}^{r}} p_{I_{1}^{s+1}} p_{L_{1}^{s+1}}\left(\pi_{I_{1}^{s}}^{*} c_{I_{2}^{s+1}, L_{1}^{s}}+\pi_{L_{1}^{s}}^{*} c_{L_{2}^{s+1}, I_{1}^{s}}\right),
$$

где $A(s, r)=\left\{I_{1}^{s}, L_{1}^{s} \in A^{s}, i_{s+1}=j_{r+1}, l_{s+1}=k_{r+1}\right\}$;

$$
\begin{aligned}
& h_{K_{1}^{r+1}, J_{1}^{r+1}}\left(M_{r}\right)=p^{2 r+2} \sum_{t=1}^{s} \sum_{B(s, r)}\left(\prod_{C\left(u_{1}, u_{2}\right)} \frac{\delta_{k_{l_{1}}, j_{l_{2}}}}{p}\left(\frac{p}{1-p}\right)^{k_{l_{1}} \oplus i_{m_{l_{1}}}}-1\right) \pi_{I_{1}^{s}}^{*} \\
& \times q_{F\left(I_{1}^{s+1} ; M_{r+1}\right)} \cdots q_{F\left(I_{t+1}^{s+t+1} ; M_{r+1}\right)} \\
& \times\left(\frac{1-p}{p}\right)^{w\left(K_{1}^{r+1} \oplus F\left(I_{1}^{s+1} ; M_{r+1}\right)\right)+w\left(J_{1}^{r+1} \oplus F\left(I_{t+1}^{s+t+1} ; M_{r+1}\right)\right)},
\end{aligned}
$$


где

$$
\begin{aligned}
B(s, r) & =\left\{I_{1}^{s+1} \in A^{s+1}, I_{s+2}^{s+t+1} \in A^{t}\right\}, \\
C\left(u_{1}, u_{2}\right) & =\left\{\left(l_{1}, l_{2}\right) \in\left\{\left(u_{1}, u_{2}\right): 1 \leqslant u_{2}<u_{1} \leqslant r+1, m_{u_{1}}=m_{u_{2}}+t\right\}\right\},
\end{aligned}
$$

и

$$
\begin{gathered}
\sigma_{K_{1}^{r+1}, J_{1}^{r+1}}^{\tilde{b}}=b_{J_{1}^{r+1}}\left(M_{r}\right)\left(\delta_{K_{1}^{r+1}, J_{1}^{r+1}}-b_{K_{1}^{r+1}}\left(M_{r}\right)\right)+h_{K_{1}^{r+1}, J_{1}^{r+1}}\left(M_{r}\right)+h_{J_{1}^{r+1}, K_{1}^{r+1}}\left(M_{r}\right) \\
+\sum_{L_{1}^{r+1}, I_{1}^{r+1} \in A^{r+1}} p^{2 r+2}\left(\frac{1-p}{p}\right)^{w\left(L_{1}^{r+1} \oplus K_{1}^{r+1}\right)+w\left(I_{1}^{r+1} \oplus J_{1}^{r+1}\right)} \varkappa_{L_{1}^{r+1}, I_{1}^{r+1}} .
\end{gathered}
$$

Лемма 4. Если $x_{t}-$ стационарная цеепь Маркова ЦМ $(s, r)$, то определяемые (9) оценки $\left\{\tilde{b}_{K_{1}^{r+1}}\left(M_{r}\right): K_{1}^{r+1} \in A^{r+1}\right\}$ являются несмещенными, состоятельными в среднеквадратическом, нормированные уклонения $\left\{\sqrt{n-s}\left(\tilde{b}_{K_{1}^{r+1}}\left(M_{r}\right)-b_{K_{1}^{r+1}}\left(M_{r}\right)\right): K_{1}^{r+1} \in A^{r+1}\right\}$ при $n \rightarrow \infty$ имеют предельное выражение для ковариаций

$$
(n-s) \operatorname{cov}\left\{\tilde{b}_{K_{1}^{r+1}}\left(M_{r}\right), \tilde{b}_{J_{1}^{r+1}}\left(M_{r}\right)\right\} \rightarrow \sigma_{K_{1}^{r+1}, J_{1}^{r+1}}^{\tilde{b}}
$$

и распределены асимптотически нормально с нулевым математическим ожиданием $и$ ковариациями (10).

Доказательство. В силу (8), (9)

$$
\begin{aligned}
\mathbf{E}\left\{\tilde{b}_{K_{1}^{r+1}}\left(M_{r}\right) t\right\} & =\frac{1}{n-s} \sum_{t=1}^{n-s} \mathbf{P}\left\{F\left(Y_{t}^{t+s-1} ; M_{r}\right)=K_{1}^{r}, y_{t+s}=k_{r+1}\right\} \\
& =b_{K_{1}^{r+1}}\left(M_{r}\right)
\end{aligned}
$$

и

$$
\begin{aligned}
& \mathbf{E}\left\{(n-s)\left(\tilde{b}_{K_{1}^{r+1}}\left(M_{r}\right)-b_{K_{1}^{r+1}}\left(M_{r}\right)\right)\left(\tilde{b}_{J_{1}^{r+1}}\left(M_{r}\right)-b_{J_{1}^{r+1}}\left(M_{r}\right)\right)\right\} \\
& =\frac{1}{n-s} \mathbf{E}\left\{v_{K_{1}^{r+1}}\left(Y_{1}^{n} ; M_{r}\right) v_{J_{1}^{r+1}}\left(Y_{1}^{n} ; M_{r}\right)\right\}-(n-s) b_{K_{1}^{r+1}}\left(M_{r}\right) b_{J_{1}^{r+1}}\left(M_{r}\right) \\
& =\delta_{K_{1}^{r+1}, J_{1}^{r+1}} b_{J_{1}^{r+1}}\left(M_{r}\right)-(n-s) b_{K_{1}^{r+1}}\left(M_{r}\right) b_{J_{1}^{r+1}}\left(M_{r}\right) \\
& +\sum_{L_{1}^{r+1}, I_{1}^{r+1} \in A^{r+1}} \sum_{\substack{t_{1}, t_{2}=1 \\
t_{1} \neq t_{2}}}^{n-s} \mathbf{P}\left\{F\left(X_{t_{1}}^{t_{1}+s} ; M_{r+1}\right)=L_{1}^{r+1}, F\left(X_{t_{2}}^{t_{2}+s} ; M_{r+1}\right)=I_{1}^{r+1}\right\} \\
& \times \mathbf{P}\left\{F\left(\Xi_{t_{1}}^{t_{1}+s} ; M_{r+1}\right)=K_{1}^{r+1} \oplus L_{1}^{r+1}, F\left(\Xi_{t_{2}}^{t_{2}+s} ; M_{r+1}\right)=J_{1}^{r+1} \oplus I_{1}^{r+1}\right\} .
\end{aligned}
$$

Используя лемму 3 (вид ковариаций для частотных статистик ЦМ $(s, r))$ из [10] и соотношения (8), (10), получаем предельное выражение для ковариаций.

Для доказательства асимптотической нормальности воспользуемся известным утверждением (см., например, [18]): случайный вектор имеет асимптотически нормальное распределение тогда и только тогда, когда произвольная линейная комбинация его компонент 
имеет асимптотически нормальное распределение. Рассмотрим вспомогательную случайную последовательность

$$
z_{t}=\sum_{K_{1}^{r+1} \in A^{r+1}} \alpha_{K_{1}^{r+1}} \delta_{F\left(Y_{t}^{t+s-1} ; M_{r}\right), K_{1}^{r}} \delta_{y_{t+s}, k_{r+1}},
$$

где $\alpha_{K_{1}^{r+1}} \in \mathbf{R}-$ произвольные коэффициенты линейной комбинации. Эта последовательность удовлетворяет условию $\varphi$-перемешивания как функция от последовательностей $\left\{x_{t}\right\},\left\{\xi_{t}\right\}$, обладающих этим свойством [19]. Кроме того, дисперсия суммы $z_{1}, z_{2}, \ldots, z_{n}$ при $n \rightarrow \infty$ бесконечно возрастает:

$$
\begin{aligned}
& \mathbf{D}\left\{\sum_{t=1}^{n-s} z_{t}\right\}=\mathbf{D}\left\{\sum_{K_{1}^{r+1} \in A^{r+1}} \alpha_{K_{1}^{r+1}} v_{K_{1}^{r+1}}\left(Y_{1}^{n} ; M_{r}\right)\right\} \\
& =(n-s)^{2} \mathbf{D}\left\{\sum_{K_{1}^{r+1} \in A^{r+1}} \alpha_{K_{1}^{r+1}} \tilde{b}_{K_{1}^{r+1}}\left(M_{r}\right)\right\} \\
& =(n-s) \sum_{K_{1}^{r+1}, J_{1}^{r+1} \in A^{r+1}} \alpha_{K_{1}^{r+1}} \alpha_{J_{1}^{r+1}} \sigma_{K_{1}^{r+1}, J_{1}^{r+1}}^{\tilde{b}} \rightarrow \infty \text {, }
\end{aligned}
$$

поэтому по центральной предельной теореме для случайных величин с $\varphi$-перемешиванием [20] получаем, что нормированная сумма

$$
(n-s)^{-1 / 2} \sum_{t=1}^{n-s} z_{t}=(n-s)^{1 / 2} \sum_{K_{1}^{r+1} \in A^{r+1}} \alpha_{K_{1}^{r+1}} \tilde{b}_{K_{1}^{r+1}}\left(M_{r}\right)
$$

распределена асимптотически нормально.

\section{4. Идентификация искаженной цепи Маркова в случае известного $p$}

Идентификация двоичной цепи Маркова при наличии аддитивных искажений заключается в оценивании параметров модели (7) и проверке гипотез о значениях ее параметров по наблюдаемой реализации $Y_{1}^{n}$.

Как уже отмечалось, из-за экспоненциальной вычислительной сложности использовать методы оценивания параметров модели (7) на основе функции правдоподобия не представляется возможным. Применим метод подстановки [21]. Согласно леммам 2, 4, оценки параметров для $\left\{\mu_{J_{1}^{r+1}}\left(M_{r}\right): J_{1}^{r+1} \in A^{r+1}\right\}$ по наблюдаемой реализации $Y_{1}^{n}$ будем находить как решение неоднородной системы $N^{r+1}$ линейных уравнений

$$
\begin{aligned}
\sum_{J_{1}^{r+1} \in A^{r+1}} \mu_{J_{1}^{r+1}}\left(M_{r}\right) & =1, \\
\sum_{J_{1}^{r+1} \in A^{r+1}} \mu_{J_{1}^{r+1}}\left(M_{r}\right) p^{r+1-w\left(J_{1}^{r+1} \oplus K_{1}^{r+1}\right)}(1-p)^{w\left(J_{1}^{r+1} \oplus K_{1}^{r+1}\right)} & =\tilde{b}_{K_{1}^{r+1}}\left(M_{r}\right),
\end{aligned}
$$


где $K_{1}^{r+1} \in A^{r+1} \backslash\{0\}^{r+1}$.

Приведем две леммы, которые понадобятся в дальнейшем при доказательстве свойств оценок, найденных из (11).

Лемма 5. Для любых $K_{1}^{r+1}, I_{1}^{r+1} \in A^{r+1}$ справедливо соотношение

$$
\left(\frac{p^{2}}{2 p-1}\right)^{r+1} \sum_{J_{1}^{r+1} \in A^{r+1}}\left(\frac{1-p}{p}\right)^{w\left(J_{1}^{r+1} \oplus K_{1}^{r+1}\right)}\left(\frac{p-1}{p}\right)^{w\left(J_{1}^{r+1} \oplus I_{1}^{r+1}\right)}=\delta_{K_{1}^{r+1}, I_{1}^{r+1}} .
$$

Доказательство. Рассмотрев два случая: $K_{1}^{r+1}=I_{1}^{r+1}$ и $K_{1}^{r+1} \neq I_{1}^{r+1}$, требуемый результат получаем прямым вычислением левой части (12).

Примем матричные обозначения

$$
V_{2}^{\prime}=\left(\begin{array}{cc}
1 & \alpha \\
\alpha & 1
\end{array}\right), \quad V_{2}^{*}=\left(\begin{array}{cc}
1 & 1 \\
\alpha & 1
\end{array}\right),
$$

$E_{2^{i}}-$ единичная матрица порядка $2^{i}$,

$$
E_{2^{i+1}}^{*}=\left(\begin{array}{c|c}
1 & 0 \ldots 0 \\
\hline 0 & \\
\vdots & \alpha E_{2^{i+1}-1}
\end{array}\right), \quad \begin{gathered}
V_{2^{i+1}}=\left(\begin{array}{c|c}
V_{2^{i}} & \alpha V_{2^{i}} \\
\hline \alpha V_{2^{i}} & \alpha V_{2^{i}}
\end{array}\right), \\
V_{2^{i+1}}^{*}=\left(\begin{array}{c|c}
E_{2^{i}}^{*} & V_{2^{i}}^{*} \\
\hline \alpha V_{2^{i}}^{\prime} & V_{2^{i}}^{\prime}
\end{array}\right),
\end{gathered}
$$

где $\alpha \in \mathbf{R}, i=1,2, \ldots$

Лемма 6. Для любого $i=1,2, \ldots$ определитель матрищьы $V_{2^{i+1}}^{*}$, заданной рекуррентной формулой (13), равен нулю тогда и только тогда, когда $\alpha= \pm 1$.

Доказательство. Используя (13) и формулу для определителя блочных матриц [22], находим, что

$$
\begin{aligned}
\left|V_{2^{i+1}}^{*}\right| & =\left|V_{2^{i}}^{\prime}\right|\left|E_{2^{i}}-\alpha E_{2^{i}}^{*}\right|\left|V_{2^{i}}^{*}\right|=\left|V_{2}^{*}\right| \prod_{k=1}^{i}\left(\left|V_{2^{k}}^{\prime}\right|\left|E_{2^{k}}-\alpha E_{2^{k}}^{*}\right|\right), \\
\left|V_{2^{i+1}}^{\prime}\right| & =\left|V_{2^{i}}^{\prime}\right|^{2}\left|E_{2^{i}}-\alpha^{2} E_{2^{i}}\right|=t\left|V_{2}^{\prime}\right|^{2^{i}} \prod_{k=1}^{i}\left(1-\alpha^{2}\right)^{2^{2 i-k}}, \\
\left|V_{2}^{*}\right| & =1-\alpha, \quad\left|V_{2}^{\prime}\right|=1-\alpha^{2}, \quad\left|E_{2^{i}}-\alpha E_{2^{i}}^{*}\right|=(1-\alpha)\left(1-\alpha^{2}\right)^{2^{i}-1},
\end{aligned}
$$

откуда получаем требуемый результат.

Введем обозначение

$$
\begin{aligned}
& \sigma_{J_{1}^{r+1}, K_{1}^{r+1}}^{\tilde{\mu}}=\varkappa_{J_{1}^{r+1}, K_{1}^{r+1}}-\mu_{J_{1}^{r+1}}\left(M_{r}\right) \mu_{K_{1}^{r+1}}\left(M_{r}\right)+\left(\frac{p}{2 p-1}\right)^{2 r+2} \\
& \times \sum \sum_{I_{1}^{r+1}, L_{1}^{r+1} \in A^{r+1}}\left(\frac{p-1}{p}\right)^{w\left(I_{1}^{r+1} \oplus J_{1}^{r+1}\right)+w\left(L_{1}^{r+1} \oplus K_{1}^{r+1}\right)} \\
& \times\left(\delta_{I_{1}^{r+1}, L_{1}^{r+1}} b_{I_{1}^{r+1}}\left(M_{r}\right)+h_{I_{1}^{r+1}, L_{1}^{r+1}}\left(M_{r}\right)\right. \\
&\left.+h_{L_{1}^{r+1}, I_{1}^{r+1}}\left(M_{r}\right)\right)
\end{aligned}
$$


где $J_{1}^{r+1}, K_{1}^{r+1} \in A^{r+1}$.

Теорема 2. Если $x_{t}-$ стациионарная ЦМ $(s, r)$ и $p \neq 1 / 2$, то оценки

$$
\tilde{\mu}_{J_{1}^{r+1}}\left(M_{r}\right)=\left(\frac{p}{2 p-1}\right)^{r+1} \sum_{K_{1}^{r+1} \in A^{r+1}} \tilde{b}_{K_{1}^{r+1}}\left(M_{r}\right)\left(\frac{p-1}{p}\right)^{w\left(J_{1}^{r+1} \oplus K_{1}^{r+1}\right)},
$$

найденные как решение системы (11), являются несмещенныли, состоятельными в среднеквадратическом; нормированные уклонения $\left\{\sqrt{n-s}\left(\tilde{\mu}_{J_{1}^{r+1}}\left(M_{r}\right)-\mu_{J_{1}^{r+1}}\left(M_{r}\right)\right)\right.$ : $\left.J_{1}^{r+1} \in A^{r+1}\right\}$ при $n \rightarrow \infty$ имеют предельное выражение для ковариащий

$$
(n-s) \operatorname{cov}\left\{\tilde{\mu}_{K_{1}^{r+1}}\left(M_{r}\right), \tilde{\mu}_{J_{1}^{r+1}}\left(M_{r}\right)\right\} \rightarrow \sigma_{K_{1}^{r+1}, J_{1}^{r+1}}^{\tilde{\mu}},
$$

и распределены асимптотически нормально с нулевым математическим ожиданием $u$ ковариациями (14).

Доказательство. Используя лемму 5, непосредственной проверкой убеждаемся, что оценки (15) являются решением системы (11). Из леммы 6 при $p \neq 1 / 2$ следует единственность решения системы (11); при этом в обозначениях (13) параметр $\alpha=(1-p) / p$.

Поскольку величины $\left\{\tilde{\mu}_{J_{1}^{r+1}}\left(M_{r}\right): J_{1}^{r+1} \in A^{r+1}\right\}$, определяемые (15), являются линейными комбинациями величин $\left\{\tilde{b}_{K_{1}^{r+1}}\left(M_{r}\right): K_{1}^{r+1} \in A^{r+1}\right\}$, из леммы 4 следуют несмещенность, состоятельность в среднеквадратическом и соотношение для ковариаций

$$
\sigma_{J_{1}^{r+1}, K_{1}^{r+1}}^{\tilde{\mu}}=\left(\frac{p}{2 p-1}\right)^{2 r+2} \sum_{I_{1}^{r+1}, L_{1}^{r+1} \in A^{r+1}}\left(\frac{p-1}{p}\right)^{w\left(I_{1}^{r+1} \oplus J_{1}^{r+1}\right)+w\left(I_{1}^{r+1} \oplus K_{1}^{r+1}\right)} \sigma_{I_{1}^{r+1}, L_{1}^{r+1}}^{\tilde{b}} .
$$

Асимптотическая нормальность следует из теоремы из [21] о непрерывном функциональном преобразовании (15) асимптотически нормальных случайных последовательностей и леммы 4.

С использованием подстановочного принципа [21] оценки $\widetilde{Q}, \widetilde{M}_{r}, \tilde{r}, \tilde{s}$ для матрицы $Q$, шаблона $M_{r}^{0}$, числа связей $r$ и порядка $s$, соответственно, можно получить с помощью формул (4)-(6), в которые вместо $\left\{\hat{\mu}_{J_{1}^{r+1}}\left(M_{r}\right): J_{1}^{r+1} \in A^{r+1}\right\}$ подставлены оценки $\left\{\tilde{\mu}_{J_{1}^{r+1}}\left(M_{r}\right): J_{1}^{r+1} \in A^{r+1}\right\}$, определяемые в (15).

Введем обозначение

$$
\begin{gathered}
\sigma_{J_{1}^{r+1}, K_{1}^{r+1}}^{\tilde{q}}=\frac{1}{\mu_{J_{1}^{r}}\left(M_{r}^{0}\right) \mu_{K_{1}^{r}}\left(M_{r}^{0}\right)}\left(\frac{p}{2 p-1}\right)^{2 r+2} \sum_{l_{1}, l_{2} \in A}\left(\delta_{j_{r+1}, l_{1}}-q_{J_{1}^{r+1}}\right)\left(\delta_{k_{r+1}, l_{2}}-q_{K_{1}^{r+1}}\right) \\
\times \sum_{I_{1}^{r+1} \in A^{r+1}}\left(\frac{p-1}{p}\right)^{w\left(I_{1}^{r} \oplus J_{1}^{r}\right)+w\left(I_{1}^{r} \oplus K_{1}^{r}\right)+w\left(i_{r+1} \oplus l_{1}\right)+w\left(i_{r+1} \oplus l_{2}\right)} b_{I_{1}^{r+1}\left(M_{r}^{0}\right),}
\end{gathered}
$$

где $J_{1}^{r+1}, K_{1}^{r+1} \in A^{r+1}$.

Теорема 3. В условиях теоремы 2 при $n \rightarrow \infty$ оченки $\widetilde{Q}, \widetilde{M}_{r}, \tilde{r}, \tilde{s}$ являются состоятельными, и при известном истинном шаблоне $M_{r}^{0}$ справедливы предельные соотношения

$$
\begin{aligned}
\mathbf{E}\left\{\tilde{q}_{J_{1}^{r+1}}\right\} & \rightarrow q_{J_{1}^{r+1},} \\
(n-s) \operatorname{cov}\left\{\tilde{q}_{K_{1}^{r+1}}, \tilde{q}_{J_{1}^{r+1}}\right\} & \rightarrow \sigma_{K_{1}^{r+1}, J_{1}^{r+1}}^{\tilde{q}},
\end{aligned}
$$


а случайные величины $\left\{\sqrt{n-s}\left(\tilde{q}_{J_{1}^{r+1}}-q_{J_{1}^{r+1}}\right), J_{1}^{r+1} \in A^{r+1}\right\}$ распределень асимптотически нормально с нулевым математическим ожиданием и ковариациями (16).

Доказательство. Используя теорему 2, теорему о непрерывном функциональном преобразовании [21], результаты из [10] и ограниченность $\tilde{q}_{J_{1}^{r+1}} \in[0,1], J_{1}^{r+1} \in A^{r+1}$, получаем доказываемое утверждение.

Следствие 3. В условиях теоремы 2, если шаблон связей $M_{r}^{0}$ известен, то при $n \rightarrow \infty$ оценка $\widetilde{Q}$ является асимптотически несмещенной и состоятельной в среднеквадратическом, причем среднеквадратическая ошибка оценивания матриць $Q$ удовлетворяет предельному соотношению

$$
\begin{aligned}
n \Delta_{n}(\tilde{Q})= & n \mathbf{E}\left\{\|\widetilde{Q}-Q\|^{2}\right\} \\
\rightarrow & \left(\frac{3 p^{2}-3 p+1}{4 p^{2}-4 p+1}\right)^{r} \sum_{J_{1}^{r}, K_{1}^{r} \in A^{r}}\left(\frac{p-p^{2}}{3 p^{2}-3 p+1}\right)^{w\left(J_{1}^{r} \oplus K_{1}^{r}\right)} \\
& \times \frac{\mu_{K_{1}^{r}}\left(M_{r}^{0}\right)}{\mu_{J_{1}^{r}}^{2}\left(M_{r}^{0}\right)}\left(\frac{2 p^{2}-2 p+1}{4 p^{2}-4 p+1}+\sum_{j_{r+1} \in A} q_{J_{1}^{r+1}}\left(q_{J_{1}^{r+1}}-2 q_{K_{1}^{r}, j_{r+1}}\right)\right) .
\end{aligned}
$$

Для доказательства следствия достаточно воспользоваться асимптотическими выражениями ковариаций (16) из теоремы 3.

Рассмотрим теперь задачу статистической проверки гипотез $H_{0}: Q=Q^{0}$, где $Q^{0}=\left(q_{J_{1}^{r+1}}^{0}\right)_{J_{1}^{r+1} \in A^{r+1}}-$ некоторая фиксированная стохастическая матрица, и $H_{1}=\bar{H}_{0}: Q \neq Q^{0}$ - альтернатива общего вида в семействе моделей (7).

Определим статистику $\rho$, полагая (с использованием (4), (15))

$$
\rho=\sum_{J_{1}^{r}, K_{1}^{r} \in A^{r}} \sum_{\substack{j_{r+1} \in D_{J_{1}^{r}}^{*} \\ k_{r+1} \in D_{K_{1}^{r}}^{*}}}(n-s)\left(\tilde{q}_{J_{1}^{r+1}}-q_{J_{1}^{r+1}}^{0}\right) \Sigma_{J_{1}^{r+1}, K_{1}^{r+1}}^{-1}\left(\tilde{q}_{K_{1}^{r+1}}-q_{K_{1}^{r+1}}^{0}\right),
$$

где

$$
D_{J_{1}^{r}}=\left\{j_{r+1} \in A: q_{J_{1}^{r+1}}^{0}>0\right\}, \quad D_{J_{1}^{r}}^{*}=D_{J_{1}^{r}} \backslash\{j\},
$$

$j$ - произвольный фиксированный элемент из $D_{J_{1}^{r}}$,

$$
\Sigma=\left(\sigma_{J_{1}^{r+1}, K_{1}^{r+1}}^{\tilde{q}}\right), \quad J_{1}^{r}, K_{1}^{r} \in A^{r}, \quad j_{r+1}, k_{r+1} \in D_{K_{1}^{r}}^{*}
$$

Рассмотрим семейство решающих правил для проверки $H_{0}, H_{1}$, основанных на (18):

$$
\text { принимается } H_{0} \text {, если } \rho \leqslant \Lambda \text {, и принимается } H_{1} \text {, если } \rho>\Lambda \text {, }
$$

где $\Lambda>0-$ некоторое пороговое значение.

Теорема 4. Если в условиях теоремы 2 справедлива гипотеза $H_{0}$, то при $n \rightarrow \infty$ статистика $\rho$, определяемая (18), имеет $\chi^{2}$-распределение с

$$
U=\sum_{J_{1}^{r} \in A^{r}}\left|D_{J_{1}^{r}}^{*}\right|
$$

степенями свободы. 
Доказательство. Используя теорему 3, теорему о распределении квадратичной формы случайных величин [23], имеющих асимптотически нормальное распределение вероятностей, получаем доказываемое утверждение.

Следствие 4. Если $\Lambda=G_{U}^{-1}(1-\varepsilon)-$ квантиль уровня $1-\varepsilon$ стандартного $\chi^{2}$-распределения с $U$ степенями свободы, то при $n \rightarrow \infty$ размер критерия (19) стремится $\kappa$ заданному уровню значимости $\varepsilon \in(0,1)$.

Следствие 5. Пусть задано семейство контигуальных альтернатив

$$
H_{1}: Q=Q^{1}
$$

гेe

$$
\begin{gathered}
Q^{1}=\left(q_{J_{1}^{r+1}}^{1}\right)_{J_{1}^{r+1} \in A^{r+1}}, \quad q_{J_{1}^{r+1}}^{1}=q_{J_{1}^{r+1}}^{0}\left(1+\frac{d_{J_{1}^{r+1}}}{\sqrt{n-s}}\right), \\
\sum_{j_{r+1} \in A} d_{J_{1}^{r+1}} q_{J_{1}^{r+1}}^{0}=0, \quad \sum_{J_{1}^{r+1} \in A^{r+1}}\left|d_{J_{1}^{r+1}}\right|>0 .
\end{gathered}
$$

Тогда при $n \rightarrow \infty$ мощчность теста (19), в котором $\Lambda=G_{U}^{-1}(1-\varepsilon)$, удовлетворяет асимптотическому соотношению

$$
w \rightarrow 1-G_{U, a}\left(G_{U}^{-1}(1-\varepsilon)\right)
$$

где $G_{U, a}(\cdot)$ - функиия нецентрального $\chi^{2}$-распределения с $U$ степенями свободы и параметром неиентральности

$$
a=\sum_{J_{1}^{r}, K_{1}^{r} \in A^{r}} \sum_{\substack{j_{r+1} \in D_{J_{1}^{r}}^{*} \\ k_{r+1} \in D_{K_{1}^{r}}^{*}}} q_{J_{1}^{r+1}}^{0} d_{J_{1}^{r+1}} \Sigma_{J_{1}^{r+1}, K_{1}^{r+1}}^{-1} q_{K_{1}^{r+1}}^{0} d_{K_{1}^{r+1}}
$$

Доказательство. В силу теоремы 3, соотношения (20) и теоремы об асимптотическом распределении суммы квадратов асимптотически нормальных случайных величин [24], получаем, что при $n \rightarrow \infty$ в случае справедливости гипотезы $H_{1}$ статистика $\rho$ имеет нецентральное $\chi^{2}$-распределение с $U$ степенями свободы и параметром нецентральности a. Таким образом, мощность критерия

$$
\begin{aligned}
w & =\mathbf{P}\left\{1-G_{U}(\rho)<\varepsilon \mid H_{1}\right\}=1-\mathbf{P}\left\{\rho \leqslant G_{U}^{-1}(1-\varepsilon) \mid H_{1}\right\} \\
& \rightarrow 1-G_{U, a}\left(G_{U}^{-1}(1-\varepsilon)\right),
\end{aligned}
$$

что совпадает с (21).

\section{5. Случай неизвестного параметра $p$}

Для идентификации модели (7) в случае неизвестного $p$ предлагаются два способа. 
Способ I. Для заданного шаблона $M_{r}$ определим расширенный шаблон

$$
M_{r^{*}}^{*}=\left(m_{1}^{*}, m_{2}^{*}, \ldots, m_{r^{*}}^{*}\right)
$$

состоящий из $r^{*}$ упорядоченных по возрастанию различных компонент, принадлежащих множеству $\left\{m_{1}, \ldots, m_{r}, s+1+m_{1}-m_{r}, \ldots, s+1+m_{r-1}-m_{r}, s+1\right\}, r+1 \leqslant r^{*} \leqslant 2 r$; селектор $F^{*}\left(J_{k}^{k+r^{*}-2} ; M_{r}\right)=\left(j_{l_{1}}, \ldots, j_{l_{r}}\right), l_{i} \in\left\{1,2 \ldots, r^{*}\right\}, m_{l_{i}}^{*}=m_{i}+(k-1)\left(s+1-m_{r}\right)$, где $i=1,2, \ldots, r, k=1,2, s^{*}=2 s+1-m_{r}$.

Лемма 7. Если $x_{t}-$ стационарная цฺепь Маркова с частичныли связями, $M_{r}=M_{r}^{0}$, mо для распределения $\left(r^{*}+1\right)$-грамм справедливо соотношение

$$
\mu_{J_{1}^{r^{*}+1}}\left(M_{r^{*}}^{*}\right)=\mu_{J_{1}^{r^{*}-1} .}\left(M_{r^{*}}^{*}\right) q_{F^{*}\left(J_{1}^{r^{*}-1} ; M_{r}^{0}\right), j_{r^{*}}} q_{F^{*}\left(J_{2}^{r^{*}} ; M_{r}^{0}\right), j_{r^{*}+1}}, \quad J_{1}^{r^{*}+1} \in A^{r^{*}+1} .
$$

Доказательство. В силу обобщенной формулы умножения вероятностей,

$$
\begin{aligned}
\mathbf{P}\left\{x_{t+m_{1}^{*}-1}=j_{1}, \ldots, x_{t+m_{r^{*}-1}^{*}}=j_{r^{*}}, x_{t+s^{*}}=j_{r^{*}+1}\right\} \\
=\mathbf{P}\left\{x_{t+s^{*}}=j_{r^{*}+1} \mid x_{t+m_{r^{*}-1}^{*}}=j_{r^{*}}, \ldots, x_{t+m_{1}^{*}-1}=j_{1}\right\} \\
\times \mathbf{P}\left\{x_{t+m_{r^{*}-1}^{*}}=j_{r^{*}} \mid x_{t+m_{r^{*}-1}^{*}-1}=j_{r^{*}-1}, \ldots, x_{t+m_{1}^{*}-1}=j_{1}\right\} \\
\quad \times \mathbf{P}\left\{x_{t+m_{r^{*}-1}^{*}-1}=j_{r^{*}-1}, \ldots, x_{t+m_{1}^{*}-1}=j_{1}\right\} .
\end{aligned}
$$

Используя (1), приходим к доказываемому утверждению.

Как следует из лемм 2, 4, 7, теорем 2, 3 и метод подстановки [21], в качестве оценки параметра $p$ можно предложить статистику

$$
\tilde{p}=\arg \min _{p \in(1 / 2,1]} \Psi(p)
$$

where

$$
\begin{aligned}
& 2 \Psi(p)=\sum_{K_{1}^{r^{*}+1} \in \Upsilon}\left(\Gamma_{K_{1}^{r^{*}+1}}(p)-\tilde{b}_{K_{1}^{r^{*}+1}}\left(M_{r^{*}}^{*}\right)\right)^{2}, \quad \Upsilon \subseteq A^{r^{*}+1}, \\
& \left.\Gamma_{K_{1}^{r^{*}+1}}(p)=p^{r^{*}+1} \sum_{J_{1}^{r^{*}+1} \in A^{r^{*}+1}} \tilde{\mu}_{J_{1}^{r^{*}-1}}\left(M_{r^{*}}^{*}\right) \tilde{q}_{F^{*}\left(J_{1}^{r^{*}-1}\right.} ; M_{r}\right), j_{r^{*}} \\
& \times \tilde{q}_{F^{*}\left(J_{2}^{r^{*}} ; M_{r}\right), j_{r^{*}+1}}\left(\frac{1-p}{p}\right)^{w\left(J_{1}^{r^{*}+1} \oplus K_{1}^{r^{*}+1}\right)},
\end{aligned}
$$

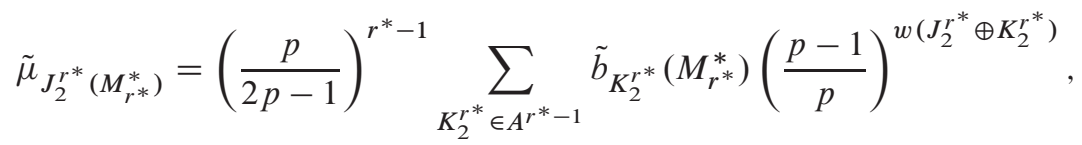

а оценку $\widetilde{Q}$ для матрицы $Q$ построить аналогично тому, как это сделано в разделе 4 данной статьи с подстановкой вместо $p$ оценки $\tilde{p}$.

Заметим, что при истинном значении параметров $p, Q$ выполняются равенства

$$
\Gamma_{K_{1}^{r^{*}+1}}(p)=b_{K_{1}^{r^{*}+1}}\left(M_{r^{*}}^{*}\right), \quad K_{1}^{r^{*}+1} \in \Upsilon .
$$


Теорема 5. Если $x_{t}$ - стационарная иепь Маркова с частичныли связями, известен шаблон связей $M_{r}^{0}$, ичелевая функичия в (22) почти наверное имеет лишь один глобальный минимум по $p \in(1 / 2,1]$, то при $n \rightarrow \infty$ оченки $\tilde{p}$, $\widetilde{Q}$, определяемые (22), являются состоятельными.

Доказательство. Используя лемму 4, теоремы 2, 3 и теорему о функциональном преобразовании сходящихся по вероятности случайных последовательностей [21], получаем требуемый результат.

Следствие 6. Если $x_{t}-$ стационарная ЦМ $(s, 1), \Upsilon=\{(0,0,0)\}-$ одноточечное множество, $p \neq 1 / 2 u$

$$
\lambda=\frac{\tilde{b}_{1}\left(\tilde{b}_{000} \tilde{b}_{0}-\tilde{b}_{00}^{2}\right)}{\tilde{b}_{0}\left(\tilde{b}_{00}^{2}+2 \tilde{b}_{00} \tilde{b}_{1}-\tilde{b}_{01} \tilde{b}_{10}\right)-\tilde{b}_{000}}>-\frac{1}{4},
$$

where $\tilde{b}_{j_{1}}=\tilde{b}_{j_{1}}\left(M_{r^{*}}^{*}\right), \tilde{b}_{j_{1} j_{2}}=\tilde{b}_{J_{1}^{2}}\left(M_{r^{*}}^{*}\right), \tilde{b}_{j_{1} j_{2} j_{3}}=\tilde{b}_{J_{1}^{3}}\left(M_{r^{*}}^{*}\right), M_{r^{*}}^{*}=(1, s+1), J_{1}^{3} \in A^{3}$, то при $n \rightarrow \infty$ состоятельной оиенкой вероятности р является статистика

$$
\tilde{p}=\frac{1}{2}(1+\sqrt{1+4 \lambda}) .
$$

Доказательство. Для цепи Маркова ЦМ $(s, 1)$ расширенный шаблон связей $M_{r^{*}}^{*}$ равен $(1, s+1), r^{*}=2$, поэтому уравнение

$$
\Gamma_{K_{1}^{r^{*}+1}}(p ; \widetilde{Q})=\tilde{b}_{K_{1}^{r^{*}+1}}\left(M_{r^{*}}^{*}\right)
$$

принимает более простой вид $p(p-1)=\lambda$ и имеет единственное решение в $(1 / 2,1]$. Это приводит к требуемому результату.

Вычисление оценки (22) может быть произведено прямым табулированием целевой функции $\Psi(p)$ по $p \in[1 / 2,1]$ с заданным шагом $h$; при этом целесообразно установить факт единственности глобального минимума функции $\Psi(p)$.

Отметим также, что в выборе $\Upsilon$ имеется некоторый произвол, позволяющий уменьшать сложность вычислений (22).

Используя минимаксное решающее правило [21], оценки для шаблона $M_{r}^{0}$, числа связей $r$ и порядка $s$ можно находить с помощью формул (5), (6), в которые вместо оценки условной энтропии $\widehat{H}\left(M_{r}\right)$ подставлена оценка $\widetilde{H}=\max \left\{\widetilde{H}_{x}, \widetilde{H}_{\xi}\right\}$, где

$$
\tilde{H}_{x}=\tilde{H}\left(M_{r}\right)=-\sum_{J_{1}^{r+1} \in A^{r+1}} \tilde{\mu}_{J_{1}^{r+1}}\left(M_{r}\right) \ln \frac{\tilde{\mu}_{J_{1}^{r+1}}\left(M_{r}\right)}{\tilde{\mu}_{J_{1}^{r}}\left(M_{r}\right)}
$$

- оценка условной энтропии ЦМ $(s, r) x_{t}$ и

$$
\tilde{H}_{\xi}=-\tilde{p} \ln \tilde{p}-(1-\tilde{p}) \ln (1-\tilde{p})
$$

- оценка удельной энтропии последовательности $\xi_{t}$, входящей в (7). 
Способ II. Этот способ основан на применении ЕМ-подхода [26]. Введем обозначения

$$
\begin{aligned}
\alpha_{J_{1}^{s}}(1) & =\pi_{J_{1}^{s}} p^{s}((1-p) / p)^{w\left(J_{1}^{s} \oplus K_{1}^{s}\right)}, \quad \beta_{J_{1}^{s}}(n-s+1)=1, \\
\alpha_{J_{1}^{s}}(t+1) & =\mathbf{P}\left\{y_{1}=k_{1}, \ldots, y_{t+s}=k_{t+s}, x_{t+1}=j_{1}, \ldots, x_{t+s}=j_{s}\right\}, \\
\beta_{J_{1}^{s}}(t) & =\mathbf{P}\left\{y_{t+s}=k_{t+s}, \ldots, y_{n}=k_{n} \mid x_{t}=j_{1}, \ldots, x_{t+s-1}=j_{s}\right\}, \\
\pi_{J_{1}^{s+1}}(t) & =\mathbf{P}\left\{x_{t}=j_{1}, \ldots, x_{t+s}=j_{s+1} \mid y_{1}=k_{1}, \ldots, y_{n}=k_{n}\right\},
\end{aligned}
$$

где $K_{1}^{n} \in A^{n}$ - реализация временного ряда (7), $J_{1}^{s+1} \in A^{s+1}, t=1, \ldots, n-s$.

Получим специальное представление для функции правдоподобия.

Теорема 6. Для любого $t \in\{1,2, \ldots, n-s\}$ справедливы равенства

$$
\begin{aligned}
\alpha_{J_{2}^{s+1}}(t+1) & =p((1-p) / p)^{k_{t+s} \oplus j_{s+1}} \sum_{j_{1} \in A} q_{F\left(J_{1}^{s} ; M_{r}^{0}\right), j_{s+1}} \alpha_{J_{1}^{s}}(t), \\
\beta_{J_{1}^{s}}(t) & =p \sum_{j_{s+1} \in A} q_{F\left(J_{1}^{s} ; M_{r}^{0}\right), j_{s+1}}((1-p) / p)^{k_{t+s} \oplus j_{s+1}} \beta_{J_{2}^{s+1}}(t+1), \\
\pi_{J_{1}^{s+1}}(t) & =\frac{p}{L(Q, p)} \alpha_{J_{1}^{s}}(t) q_{F\left(J_{1}^{s} ; M_{r}^{0}\right), j_{s+1}}((1-p) / p)^{k_{t+s} \oplus j_{s+1}} \beta_{J_{2}^{s+1}}(t+1),
\end{aligned}
$$

где $J_{1}^{s+1} \in A^{s+1}$.

Доказательство. Прямым вычислением на основе (7) получаем, что

$$
\begin{aligned}
\alpha_{J_{2}^{s+1}}(t+1)= & \mathbf{P}\left\{y_{t+s}=k_{t+s} \mid Y_{1}^{t+s-1}=K_{1}^{t+s-1}, X_{t+1}^{t+s}=J_{2}^{s+1}\right\} \\
& \times \mathbf{P}\left\{Y_{1}^{t+s-1}=K_{1}^{t+s-1}, X_{t+1}^{t+s}=J_{2}^{s+1}\right\} \\
= & \mathbf{P}\left\{\xi_{t+s}=k_{t+s} \oplus j_{s+1}\right\} \sum_{j_{1} \in A} \mathbf{P}\left\{Y_{1}^{t+s-1}=K_{1}^{t+s-1}, X_{t}^{t+s}=J_{1}^{s+1}\right\}, \\
\beta_{J_{1}^{s}}(t)= & \frac{1}{\mathbf{P}\left\{X_{t}^{t+s-1}=J_{1}^{s}\right\}} \sum_{j_{s+1} \in A} \mathbf{P}\left\{Y_{t+s}^{n}=K_{t+s}^{n}, X_{t}^{t+s}=J_{1}^{s+1}\right\} \\
= & \sum_{j_{s}+1 \in A} \mathbf{P}\left\{x_{t+s}=j_{s+1} \mid X_{t}^{t+s-1}=J_{1}^{s}\right\} \\
& \times \mathbf{P}\left\{y_{t+s}=k_{t+s} \mid X_{t}^{t+s}=J_{1}^{s+1}\right\} \\
& \times \mathbf{P}\left\{Y_{t+s+1}^{n}=K_{t+s+1}^{n} \mid y_{t+s}=k_{t+s}, X_{t}^{t+s}=J_{1}^{s+1}\right\}, \\
\pi_{J_{1}^{s+1}(t)=} & \quad 1 \\
& \left.\quad \times \quad Y_{1}^{n}=K_{1}^{n}\right\}\left\{x_{t+s}=j_{s+1} \mid X_{1}^{t+s-1}=K_{1}^{t+s-1}, X_{t}^{t+s-1}=J_{1}^{s}\right\} \\
& \times \mathbf{P}\left\{y_{t+s}=k_{t+s} \mid X_{t}^{t+s}=J_{1}^{s+1}, Y_{1}^{t+s-1}=K_{1}^{t+s-1}\right\} \\
& \times \mathbf{P}\left\{Y_{t+s+1}^{n}=K_{t+s+1}^{n} \mid X_{t}^{t+s}=J_{1}^{s+1}, Y_{1}^{t+s}=K_{1}^{t+s}\right\},
\end{aligned}
$$

откуда следуют доказываемые соотношения.

Функцию правдоподобия $L(Q, p)$, определенную в лемме 3 , можно вычислить по 
формуле

$$
\begin{aligned}
L(Q, p)= & \sum_{J_{1}^{s} \in A^{s}} \mathbf{P}\left\{Y_{1}^{n}=K_{1}^{n}, X_{t}^{t+s-1}=J_{1}^{s}\right\} \\
= & \sum_{J_{1}^{s} \in A^{s}} \mathbf{P}\left\{Y_{1}^{t+s-1}=K_{1}^{t+s-1}, X_{t}^{t+s-1}=J_{1}^{s}\right\} \\
& \quad \times \mathbf{P}\left\{Y_{t+s}^{n}=K_{t+s}^{n} \mid Y_{1}^{t+s-1}=K_{1}^{t+s-1}, X_{t}^{t+s-1}=J_{1}^{s}\right\} \\
= & \sum_{J_{1}^{s} \in A^{s}} \alpha_{J_{1}^{s}}(t) \beta_{J_{1}^{s}}(t), t \in\{1,2, \ldots, n-s+1\} .
\end{aligned}
$$

Аналогичный подход для вычисления функции правдоподобия использовался в рабо$\operatorname{Tax}[12,13]$.

На основании теоремы 6 строится алгоритм оценивания параметров модели (7).

(1) Выбираем начальные значения $Q^{(0)}, p^{(0)}$ и по (24) вычисляем $L^{(0)}=L\left(Q^{(0)}, p^{(0)}\right)$; целесообразно использовать матрицу

$$
Q^{(0)}=\left(\tilde{b}_{J_{1}^{r+1}}\left(M_{r}^{0}\right) / \tilde{b}_{J_{1}^{r}} \cdot\left(M_{r}^{0}\right)\right)_{J_{1}^{r+1} \in A^{r+1}} .
$$

(2) Пусть для $i \geqslant 0$ известны приближения $i$-го шага $Q^{(i)}, p^{(i)}$, тогда находим

$$
\begin{aligned}
& q_{J_{1}^{r+1}}^{(i+1)}=\mu_{J_{1}^{r+1}}^{(i)}\left(M_{r}^{0} / \mu_{J_{1}^{r}}^{(i)}\left(M_{r}^{0}\right),\right. \\
& p^{(i+1)}=\sum_{t=1}^{n-s} \sum_{I_{1}^{s} \in A^{s}} \pi_{I_{1}^{s}, k_{t+s}}^{(i)}(t) /(n-s),
\end{aligned}
$$

где

$$
\mu_{J_{1}^{r+1}}^{(i)}\left(M_{r}^{0}\right)=\sum_{t=1}^{n-s} \sum_{I_{1}^{s} \in A^{s}} \delta_{F\left(I_{1}^{s} ; M_{r}^{0}\right), J_{1}^{r}} \pi_{I_{1}^{s}, j_{r+1}}^{(i)}(t)
$$

Затем согласно (24) вычисляем значение функции $L^{(i+1)}=L\left(Q^{(i+1)}, p^{(i+1)}\right)$.

(3) Если $L^{(i+1)}-L^{(i)}<\varepsilon$, то “останов” и $\widetilde{Q}=Q^{(i+1)}, \tilde{p}=p^{(i+1)}$, иначе возвращаемся к шагу 2 при $i=i+1 ; \varepsilon>0$ - параметр “останова” итерационного процесса.

Заметим, что величины $\alpha_{J_{1}^{s}}(t), \beta_{J_{1}^{s}}(t), \pi_{J_{1}^{s+1}}(t)$ могут достигать значения столь близкие к нулю, что их нельзя обрабатывать на компьютере. Для преодоления этой трудности предлагается проводить нормировку указанных величин [13].

Способ II на основе ЕМ-подхода по сравнению со способом I имеет два недостатка: увеличение порядка $s$ цепи Маркова с частичными связями ведет к экспоненциальному росту времени работы алгоритма оценивания параметров $O\left(n 2^{s}\right)$; отсутствуют теоретические результаты о состоятельности и несмещенности оценок. 
Таблица 1. Зависимость оценки $\Delta_{n}(\tilde{p})$ от длительности наблюдения

\begin{tabular}{c|ccccccc}
$n \cdot 10^{-6}$ & 10 & 15 & 20 & 25 & 30 & 35 & 40 \\
\hline$p=0,675$ & 0,00808 & 0,00629 & 0,00535 & 0,00462 & 0,00418 & 0,00386 & 0,00354 \\
$p=0,705$ & 0,00358 & 0,00280 & 0,00232 & 0,00199 & 0,00175 & 0,00156 & 0,00142
\end{tabular}

Таблица 2. Зависимость оценок $\Delta_{n}(\tilde{p}), \gamma$ от $n$

\begin{tabular}{c|ccccccc}
$n \cdot 10^{-4}$ & 10 & 15 & 20 & 25 & 30 & 35 & 40 \\
\hline$\Delta_{n}(\tilde{p})$ & 0.0092 & 0.0072 & 0.0064 & 0.0060 & 0.0051 & 0.0043 & 0.0043 \\
$\gamma$ & 0.3125 & 0.1964 & 0.1399 & 0.1161 & 0.0625 & 0.0357 & 0.0446
\end{tabular}

\section{6. Результаты компьютерных экспериментов}

Пример 1. Исследовалась двоичная цепь Маркова ЦМ $(256,3)$ с шаблоном $M_{r}^{0}=(1,7,90)$ и матрицей $Q$ (в транспонированной форме)

$$
Q^{\prime}=\left(\begin{array}{llllllll}
0,279 & 0,758 & 0,364 & 0,882 & 0,415 & 0,656 & 0,707 & 0,324 \\
0,721 & 0,242 & 0,636 & 0,118 & 0,585 & 0,344 & 0,293 & 0,676
\end{array}\right)
$$

при наличии аддитивных искажений (7) с параметром $p \in\{0,675,0,705\}$.

В табл. 1 представлена зависимость экспериментального значения $\Delta_{n}(\tilde{p})=\mathbf{E}\{|\tilde{p}-p|\}$ отклонения оценки $\tilde{p}$, найденной способом I (при $h=10^{-3}$ ), от длительности наблюдения $n$. Компьютерное моделирование проводилось по методу Монте-Карло (при числе прогонов, равном $10^{3}$ ).

На рис. 1 представлена зависимость среднеквадратической ошибки оценивания матрицы $Q$ от длительности наблюдения $n\left(a-\right.$ экспериментальные значения $\Delta_{n}(\widetilde{Q})$ и $99 \%$ доверительные границы при неизвестном $p=0,675 ; b$ - экспериментальные, теоретические значения среднеквадратической ошибки оценивания (17) и 99\% доверительные границы при известном $p=0,675 ; c$ - экспериментальные, теоретические значения среднеквадратической ошибки оценивания (17) и 99\% доверительные границы при известном $p=0,705)$. Экспериментальные значения среднеквадратической ошибки оценивания получены с помощью компьютерного моделирования по методу Монте-Карло.

Из рассмотренного примера видно, что экспериментальные значения среднеквадратической ошибки оценивания согласуются с теоретическими (17); чем ближе параметр $p$ к $1 / 2$, тем ниже точность идентификации цепи Маркова при наличии искажений.

Для оценивания параметра $p$ матрицы $Q$ по одной реализации длительности $n=30 \cdot 10^{6}$ на Intel Celeron CPU $1.70 \mathrm{GHz}$ затрачивалось примерно 13,43 с.

Пример 2. Исследовалась двоичная цепь Маркова ЦМ $(32,3)$ с шаблоном $M_{r}^{0}=(1,4,27)$ и матрицей $Q$ (в транспонированной форме)

$$
Q^{\prime}=\left(\begin{array}{llllllll}
0,649 & 0,881 & 0,195 & 0,731 & 0,395 & 0,695 & 0,304 & 0,642 \\
0,351 & 0,119 & 0,805 & 0,269 & 0,605 & 0,305 & 0,696 & 0,358
\end{array}\right)
$$

при наличии аддитивных искажений (7) с параметром $p=0,75$. В предположении, что $p$ и $M_{r}^{0}$ неизвестны, исследовалась зависимость экспериментальных значений $\Delta_{n}(\tilde{p})$, $\gamma=\mathbf{P}\left\{\widetilde{M}_{r} \neq M_{r}^{0}\right\}$, найденных методом Монте-Карло (при числе прогонов, равном $10^{3}$ ). Численные результаты, представленные в табл. 2, демонстрируют состоятельность оценки $\tilde{p}$, найденной первым способом при $h=(1 / 2) \cdot 10^{-2}$, и оценки $\widetilde{M}_{r}$, построенной с помощью минимаксного решающего правила. 
(a)

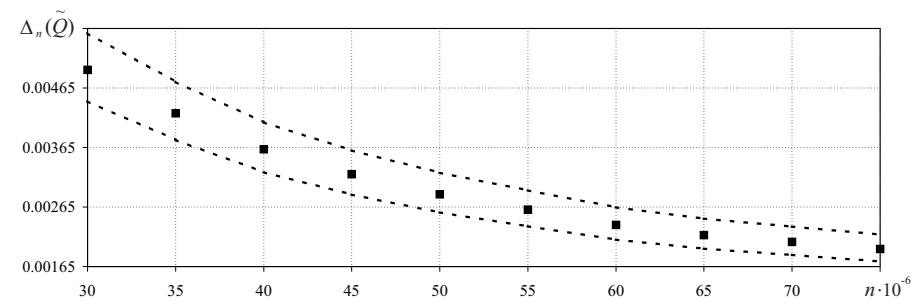

(b)

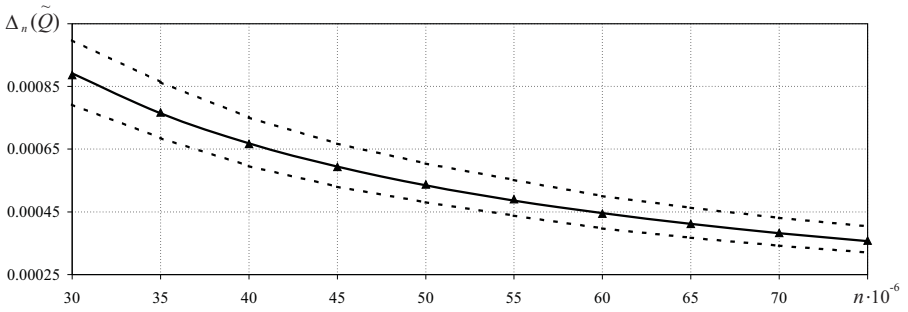

(c)

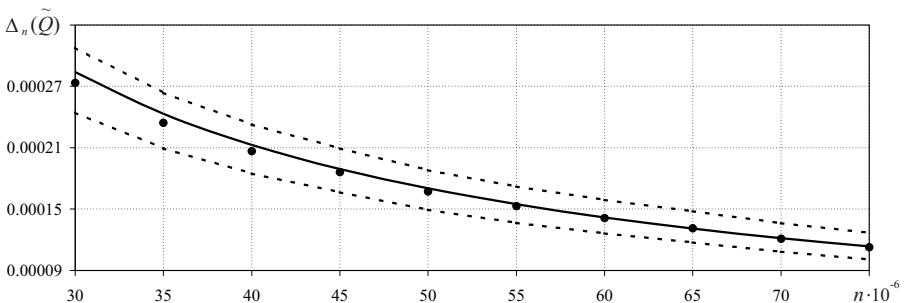

Рис. 1. Зависимость среднеквадратической ошибки оценивания от длительности наблюдения

Таблица 3. Зависимость оценок $\Delta_{n}(\tilde{p}), \Delta_{n}(\tilde{Q})$ от $n$

\begin{tabular}{cc|ccccccc}
\multicolumn{2}{c|}{$n \cdot 10^{-4}$} & 1 & 2 & 3 & 4 & 5 & 6 & 7 \\
\hline$\Delta_{n}(\tilde{p})$ & I & 0.0336 & 0.0220 & 0.0190 & 0.0159 & 0.0145 & 0.0131 & 0.0127 \\
& II & 0.0795 & 0.0710 & 0.0566 & 0.0450 & 0.0342 & 0.0283 & 0.0250 \\
\hline$\Delta_{n}(\widetilde{Q})$ & I & 0.0340 & 0.0174 & 0.0124 & 0.0095 & 0.0076 & 0.0064 & 0.0061 \\
& II & 0.0743 & 0.0626 & 0.0458 & 0.0324 & 0.0205 & 0.0148 & 0.0118
\end{tabular}

Пример 3. Для двоичной цепи Маркова ЦМ $(6,2)$ с шаблоном $M_{r}^{0}=(1,6)$ и матрицей $Q$ (в транспонированной форме)

$$
Q=\left(\begin{array}{llll}
0,398 & 0,714 & 0,656 & 0,183 \\
0,602 & 0,286 & 0,344 & 0,817
\end{array}\right)
$$

при наличии аддитивных искажений (7) с параметром $p=0,825$ исследована точность оценок $\tilde{p}, \tilde{Q}$, найденных двумя способами (способы I, II). В табл. 3 представлена зависимость экспериментальных значений $\Delta_{n}(\tilde{p}), \Delta_{n}(\tilde{Q})$, найденных методом Монте-Карло (при числе прогонов, равном 250), от длительности наблюдения $n$ при $h=10^{-3}$ для способа I, $p^{(0)}=0,9$ и $\varepsilon=0,1$ для способа II.

Полученные экспериментальные результаты иллюстрируют два факта:

(1) состоятельность оценок $\tilde{p}, \widetilde{Q}$;

(2) способ I более точно оценивает параметры, чем способ II на основе ЕМ-подхода.

Авторы глубоко благодарны А. М. Зубкову за уточнения постановок задач и замечания, способствовавшие улучшению статьи. 


\section{Список литературы}

1. Алферов А. П., Зубов А. Ю., Кузьмин А. С., Черемушкин А. В., Основы криптографии. Гелиос, Москва, 2001.

2. Дуб Дж., Вероятностные прочессы. ИЛ, Москва, 1956.

3. Зубков А. М., Датчики псевдослучайных чисел и их применения. В сб.: Труды II Международной научной конф. «Математика и безопасность информационных технологий». Изд-во МГУ, Москва, 2003, с. 200-206.

4. Харин Ю. С., Берник В. И, Матвеев Г. В., Агиевич С. В., Математические и компьютерные основы криптологии. Новое знание, Минск, 2003.

5. Максимов Ю. И., О цепях Маркова, связанных с двоичными регистрами сдвига со случайными элементами. Труды по дискретной математике (1997) 1, 203-220.

6. Jacobs P. A., Lewis P. A. W., Discrete time series generated by mixtures. I: Correlational and runs properties. J. Royal Stat. Soc., Ser. B (1978) 40, 94-105.

7. Raftery A. E., A model for high-order Markov chains. J. Royal Statist. Soc. (1985) 47, 528-539.

8. Bühlmann P., Wyner A., Variable length Markov chains. Ann. Stat. (1999) 27, №2, 480-513.

9. Харин Ю. С., Цепи Маркова с $r$-частичными связями и их статистическое оценивание. Докл. НАН Беларуси (2004) 48, №1, 40-44.

10. Харин Ю. С., Петлицкий А. И., Цепь Маркова $s$-го порядка с $r$ частичными связями и статистические выводы о ее параметрах. Дискретная математика (2007) 19, №2, 109-130.

11. Sugimoto S., Ishizuka I., Identification and estimation algorithms for a Markov chain plus AR process. Proc. IEEE ICASSP'83 8, 247-250.

12. Rabiner L. R., A tutorial on hidden Markov models and selected applications in speech recognition. Proc. IEEE (1989) 77, 257-286.

13. Berchtold A., The double chain Markov model. Commun. Stat., Theory Methods (1999) 28, №11, 2569-2589.

14. Кемени Дж. Г., Снелл Дж. Л., Конечные ичепи Маркова. Наука, Москва, 1970.

15. Харин Ю. С., Петлицкий А. И., Об оценивании порядка цепи Маркова с частичными связями. В сб.: Труды ИСТ'2006, 1, с. 156-161.

16. Csiszar I., Shields P. C., The consistency of the BIC Markov order estimator. Ann. Stat. (2000) 28, №6, 1601-1619.

17. Петлицкий А. И., Харин Ю. С., Статистический анализ двоичной цепи Маркова с частичными связями при наличии аддитивных искажений. Весиі НАН Беларусі, Сер. фiз.-мат. навук (2008) №4, 30-36.

18. Ширяев А. Н., Вероятность. Наука, Москва, 1989.

19. Bradley R. C., Basic properties of strong mixing conditions. A survey and some open questions. Probab. Surv. (2005) 2, 107-144.

20. Ибрагимов И. А., Линник Ю. В., Независимые и стаџионарно связанные величины. Наука, Москва, 1965.

21. Боровков А. А., Математическая статистика. Наука, Москва, 1984.

22. Гантмахер Ф. Р., Теория матрии. Наука, Москва, 1966.

23. Ивченко Г. И., Медведев Ю. И., Математическая статистика. Высшая школа, Москва, 1984.

24. Большев Л. Н., Смирнов Н. В., Таблицы математической статистики. Наука, Москва, 1983.

25. Самарский А. А., Гулин А. В., Численныле методы. Наука, Москва, 1989.

26. Gourieroux C., Monfort A., Statistics and econometric models: General concepts, estimation, prediction, and algorithms, 1. Cambridge Univ. Press, Cambridge, 1995. 